\title{
Dilated Thin-Walled Blood and Lymphatic Vessels in Human Endometrium: A Potential Role for VEGF-D in Progestin-Induced Break-Through Bleeding
}

\author{
Jacqueline F. Donoghue ${ }^{1 \times a}$, C. Jay McGavigan ${ }^{1 x b}$, Fiona L. Lederman ${ }^{2}$, Leonie M. Cann², Lulu Fu ${ }^{2}$, Eva \\ Dimitriadis ${ }^{3}$, Jane E. Girling ${ }^{2}$, Peter A. W. Rogers ${ }^{2 *}$
}

1 Centre for Women's Health Research, Department of Obstetrics and Gynaecology and Monash Institute for Medical Research, Monash University, Clayton, Victoria, Australia, 2 Gynaecology Research Centre, Department of Obstetrics and Gynaecology, The University of Melbourne, Royal Women's Hospital, Melbourne, Victoria, Australia, 3 Embryo Implantation Laboratory, Prince Henry's Institute for Medical Research, Monash Medical Centre, Clayton, Victoria, Australia

\begin{abstract}
Progestins provide safe, effective and cheap options for contraception as well as the treatment of a variety of gynaecological disorders. Episodes of irregular endometrial bleeding or breakthrough bleeding (BTB) are a major unwanted side effect of progestin treatment, such that BTB is the leading cause for discontinued use of an otherwise effective and popular medication. The cellular mechanisms leading to BTB are poorly understood. In this study, we make the novel finding that the large, dilated, thin walled vessels characteristic of human progestin-treated endometrium include both blood and lymphatic vessels. Increased blood and lymphatic vessel diameter are features of VEGF-D action in other tissues and we show by immunolocalisation and Western blotting that stromal cell decidualisation results in a significant increase in VEGF-D protein production, particularly of the proteolytically processed $21 \mathrm{kD}$ form. Using a NOD/scid mouse model with xenografted human endometrium we were able to show that progestin treatment causes decidualisation, VEGF-D production and endometrial vessel dilation. Our results lead to a novel hypothesis to explain BTB, with stromal cell decidualisation rather than progestin treatment per se being the proposed causative event, and VEGF-D being the proposed effector agent.
\end{abstract}

Citation: Donoghue JF, McGavigan CJ, Lederman FL, Cann LM, Fu L, et al. (2012) Dilated Thin-Walled Blood and Lymphatic Vessels in Human Endometrium: A Potential Role for VEGF-D in Progestin-Induced Break-Through Bleeding. PLoS ONE 7(2): e30916. doi:10.1371/journal.pone.0030916

Editor: Marc Tjwa, University of Frankfurt - University Hospital Frankfurt, Germany

Received September 16, 2011; Accepted December 24, 2011; Published February 17, 2012

Copyright: (ㄷ 2012 Donoghue et al. This is an open-access article distributed under the terms of the Creative Commons Attribution License, which permits unrestricted use, distribution, and reproduction in any medium, provided the original author and source are credited.

Funding: JFD was supported by an Australian Postgraduate Award. This study was funded by National Health and Medical Research Council Grant No. 384195 to PAWR and JEG. ED salary paid by National Health and Medical Research Council Career Development Award Grant No. 550911. PAWR salary paid by National Health and Medical Research Council Fellowship Grant No. 334063. The funders had no role in study design, data collection and analysis, decision to publish, or preparation of the manuscript.

Competing Interests: The authors have declared that no competing interests exist.

*E-mail: parogers@unimelb.edu.au

wa Current address: Oncogenic Signalling Laboratory, Monash Institute for Medical Research, Monash University, Clayton, Victoria, Australia ab Current address: Department of Obstetrics and Gynaecology, Flinders Medical Centre, Bedford Park, South Australia, Australia

\section{Introduction}

Progestins provide safe, effective and cheap options for contraception as well as the treatment of a variety of gynaecological disorders [1]. Although originally developed as a contraceptive, the levonorgestrel-releasing intra-uterine system (LNG-IUS) is also highly effective at controlling excessive menstrual blood loss in the majority of women treated [2]. Progestins are also used clinically in a number of other situations such as to provide relief from the symptoms of endometriosis by suppressing growth of the ectopic endometrium [3]. However, episodes of irregular endometrial bleeding or breakthrough bleeding (BTB) are a major unwanted side effect of progestin treatment, such that BTB is the leading cause for discontinued use of an otherwise effective and popular medication [4,5]. Endometrial breakthrough bleeding is defined as any irregular or unpredictable bleeding that is not part of the normal menstrual process. Unlike menstrual bleeding, which occurs primarily from the spiral arterioles in response to falling levels of estrogen and progesterone [6], BTB occurs from the endometrial capillaries and smaller vessels [7].
Understanding of the cellular mechanisms underlying BTB remains elusive because of both the range of hormonal conditions under which it can occur and the high degree of variability between women in terms of their endometrial response to exogenous hormones and their susceptibility to BTB. Thus, while administration of exogenous progestins will increase the incidence of BTB, the link between hormones and BTB is not direct. Numerous studies over the past 20 years have been undertaken with the aim of developing a better understanding of the local endometrial mechanisms responsible for BTB. Some of factors that have been proposed as playing a role in BTB include endometrial epithelial integrity [8], altered MMP levels [9], altered leucocyte populations [10], reduced vascular pericyte coverage [11,12], and increased vascular fragility [13]. Despite this work, widespread consensus as to mechanisms that might be responsible for BTB does not exist yet within the field.

Decidualisation is a hormonally regulated cellular differentiation process that occurs in the endometrial stroma of most mammals prior to and during placentation. It is accompanied by significant vascular remodelling of both blood and lymphatic 
vessels [14,15] as part of the process of ensuring an adequate blood supply to the placenta. In humans, decidualisation commences during the mid-late secretory phase under the influence of luteal progesterone regardless of whether embryo implantation has occurred. However, if implantation does not occur, circulating progesterone levels drop rapidly and the decidualised tissue is shed a few days later during menstruation.

Endometrium exposed to progestins undergoes a well characterised series of morphological changes that includes stromal cell pseudo-decidualisation, epithelial cell regression [16] and the appearance of abnormally dilated and thin-walled endometrial vessels $[7,17,18,19,20,21,22]$. While it has been proposed that the abnormally dilated, thin walled vessels found in progestin-exposed endometrium may contribute to BTB through increased vascular fragility [13], there is currently a lack of information characterizing these vessels, and little insight as to the mechanisms behind their formation. If these dilated, thin-walled vessels do play a role in BTB, a better understanding of how they are formed may assist in identifying new avenues for treating this troublesome side-effect of progestin treatment.

The overall aim of this study was to investigate potential mechanisms by which progestin administration results in the formation of abnormally dilated and thin-walled endometrial vessels.

We were able to demonstrate that a significant proportion of the dilated, thin walled vessels in progestin treated human endometrium are lymphatics and not blood vessels. Both lymphatic and blood vessels show marked increases in diameter following progestin treatment, while lymphatics in the functional layer are the only vessels to show a moderate increase in number. Increased blood and lymphatic vessel diameter, but not number, are features of VEGF-D action in other tissues [23] and we show by immunolocalisation and Western blotting that stromal cell decidualisation results in a significant increase in VEGF-D protein production, particularly of the biologically active $21 \mathrm{kD}$ form. We then use a NOD/scid mouse model with xenografted human endometrium to show that progestin treatment causes decidualisation, VEGF-D production and endometrial vessel dilation. Our results lead to a novel hypothesis to explain $\mathrm{BTB}$, with stromal cell decidualisation rather than progestin treatment per se being the proposed causative event, and VEGF-D being the proposed effector agent.

\section{Results}

Endometrial Blood and Lymphatic Vessels have Increased Diameters in Women Exposed to Intra-Uterine

\section{Levonorgestrel}

Full-thickness endometrial sections from women with heavy menstrual bleeding who had either been treated with a levonorgestrel intrauterine system (LNG-IUS) $(\mathrm{N}=16)$ or who were untreated controls $(\mathrm{N}=16)$ [22], were immunostained with antibodies to CD31 (labels both lymphatic and blood endothelial cells) or D2-40 (labels lymphatic endothelial cells only). Blood vessel counts were made from CD31 immunostained sections by subtracting the D2-40 immunostained vessel count made on a serial section. Endometrial blood and lymphatic vessels were readily identifiable in all sections following immunostaining (Fig. 1). Most of the microvessels were of normal appearance in both the LNG-IUS treated tissue and the controls. However, in the levonorgestrel treated tissue, but not the control tissue, large, thin walled vessels were also observed. Immunohistochemistry with D2-40 revealed that some of these vessels were lymphatics. In LNG-IUS treated tissue, the large or dilated lymphatic and blood vessels appeared to be mostly located in the functional layer, although a few were also seen in the basal layer.

Using 2-way ANOVA, the vessel cross sectional area of the 5 largest blood vessels was significantly greater in endometrium from

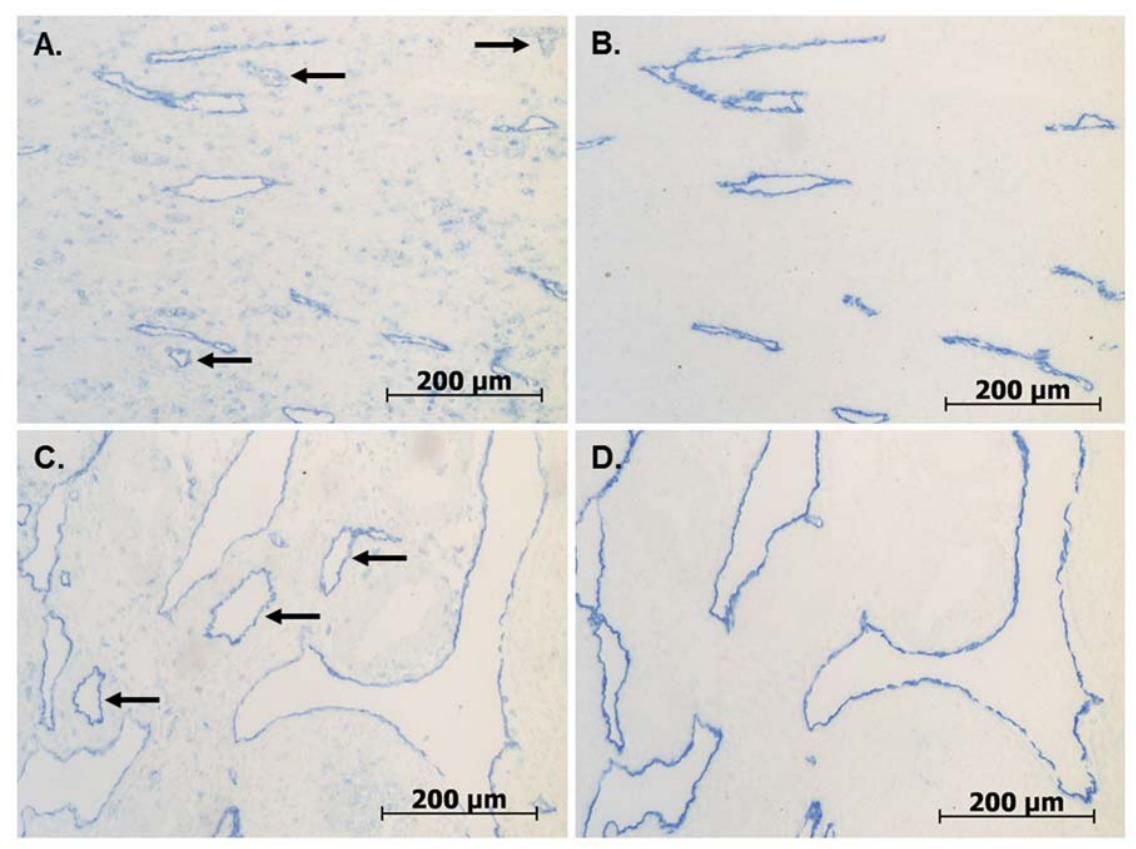

Figure 1. Progestin-induced endometrial blood and lymph vessel dilatation. Enlarged blood and lymphatic vessels are present in the endometrium of women treated with LNG-IUS prior to hysterectomy for heavy menstrual bleeding. $(A-B)$ Untreated control samples. (C-D) LNG-IUS treated samples. Sections have been immunostained with CD31 $(A, C)$, which labels both blood and lymphatic endothelial cells, or D2-40 $(B, D)$, which labels lymphatic endothelial cells only. Black arrows: blood vessels.

doi:10.1371/journal.pone.0030916.g001 
women treated with LNG-IUS compared to controls $\left(\mathrm{F}_{(1,59)}=\right.$ 12.693, $P=0.001$ ) (Fig. 2A). Tukey's posthoc tests showed that this observation was true in the functional layer (Control: $1.0 \times$ $10^{-3} \pm 3.8 \times 10^{-4} \mathrm{~mm}^{2}$; LNG-IUS: $3.5 \times 10^{-3} \pm 9.7 \times 10^{-4} \mathrm{~mm}^{2}$; $P=0.026$ ) but not the basal layer (Control: $1.2 \times 10^{-3} \pm 3.7 \times$ $10^{-4} \mathrm{~mm}^{2}$; LNG-IUS: $\left.4.6 \times 10^{-3} \pm 1.4 \times 10^{-3} \mathrm{~mm}^{2} ; P=0.165\right)$. A similar analysis of the five largest lymphatic vessels showed a significant increase in area for LNG-IUS versus control treated tissues $\left(\mathrm{F}_{(1,60)}=19.569, P=0.001\right)$ (Fig. 2B). This was the case in both the functional layer (Control: $6.4 \times 10^{-4} \pm 2.3 \times 10^{-4} \mathrm{~mm}^{2}$; LNG-IUS: $\left.3.8 \times 10^{-3} \pm 9.8 \times 10^{-4} \mathrm{~mm}^{2} ; \quad P=0.01\right)$ and also the basal layer (Control: $1.0 \times 10^{-3} \pm 3.1 \times 10^{-4} \mathrm{~mm}^{2}$; LNG-IUS: $\left.3.2 \times 10^{-3} \pm 8.8 \times 10^{-4} \mathrm{~mm}^{2} P=0.021\right)$.

In contrast to vessel cross sectional areas, there was no difference in endometrial blood vessel density $\left(\mathrm{F}_{(1,53)}=1.08\right.$, $P=0.304$ or lymph vessel density $\left(\mathrm{F}_{(1,63)}=2.384, P=0.128\right)$ between control or LNG-IUS treated tissues (Fig. 3). Also, there was no overall significant difference in vessel density between the functional layer vs. the basal layer for blood vessels (Functional Layer: Control: $42 \pm 6.9$ vessels $/ \mathrm{mm}^{2}$; LNG-IUS: $45 \pm 10.7$ vessels $/ \mathrm{mm}^{2}$ Basal Layer: Control: $31 \pm 4.8$ vessels $/ \mathrm{mm}^{2}$; LNGIUS: $39 \pm 8.6$ vessels $\left./ \mathrm{mm}^{2} ; \mathrm{F}_{(1,53)}=0.342, P=0.562\right)$ or lymphatics (Functional Layer: Control: $9 \pm 2.5$ vessels $/ \mathrm{mm}^{2}$; LNG-IUS: $23 \pm 4.8$ vessels $/ \mathrm{mm}^{2}$ Basal Layer: Control: $24 \pm 5.0$ vessels $/ \mathrm{mm}^{2}$; LNG-IUS: $20 \pm 2.6$ vessels $\left./ \mathrm{mm}^{2} ; \mathrm{F}_{(1,63)}=2.779, P=0.101\right)$.

\section{A. Blood vessel area}

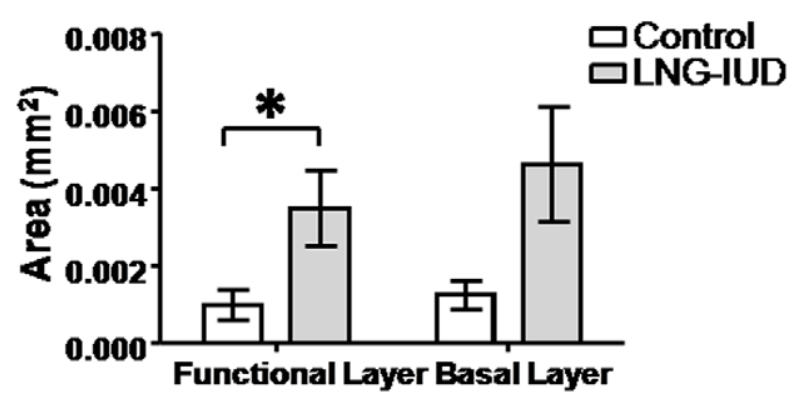

B. Lymphatic vessel area

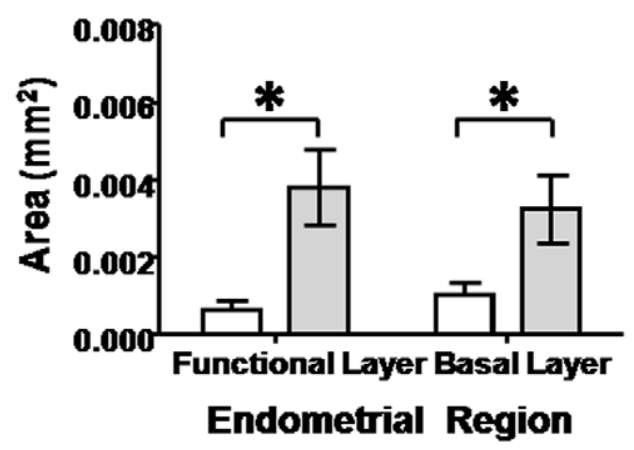

Figure 2. Increased blood and lymphatic vessel area after progestin exposure. Blood and lymphatic vessel cross sectional areas are increased in endometrium from women treated with LNG-IUS prior to hysterectomy for heavy menstrual bleeding. (A) The mean area of the five largest blood vessels in the functional layer and the basal layer in untreated control (white bars) and LNG-IUS treated (grey bars) endometrium. (B) The mean area of the five largest lymphatic vessels in the functional layer and the basal layer of untreated control (white bars) and LNG-IUS treated (grey bars) endometrium. Columns represent means \pm SE. ${ }^{*}, P<0.05$.

doi:10.1371/journal.pone.0030916.g002

\section{A. CD31}

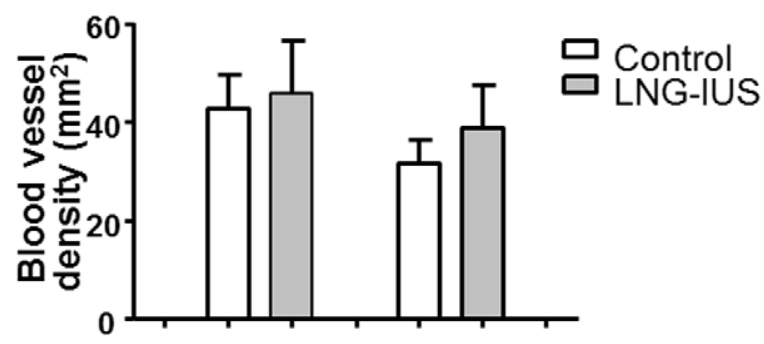

\section{B. D240}

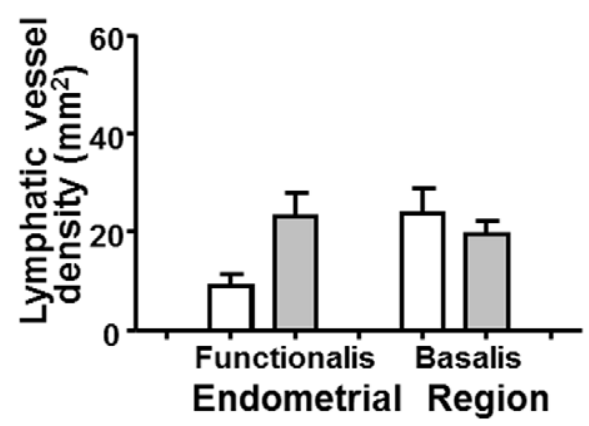

Figure 3. Endometrial blood and lymphatic vessel density after progestin exposure. Blood and lymphatic vessel densities do not change in endometrium from women treated with LNG-IUS prior to hysterectomy for heavy menstrual bleeding. (A) Vascular density of blood vessels in untreated control (white bars) and LNG-IUS treated (grey bars) samples of the functional layer and the basal layer. (B) Lymphatic vessel density in untreated and LNG-IUS treated endometrial samples. Columns represent means $\pm \mathrm{SE}$. ${ }^{*}, P<0.05$. doi:10.1371/journal.pone.0030916.g003

\section{Human Endometrial Stromal Cells Show Increased Expression of VEGF-D Following Decidualisation}

Increased blood and lymphatic vessel diameters are a recognised consequence of elevated tissue expression of VEGF-D [23]. Immunohistochemical and Western blotting techniques were used to investigate whether VEGF-D protein levels are increased in decidualised endometrial stromal cells. Focal areas of predecidualisation were common in endometrium treated with LNGIUS $(N=16)$ but not in endometrium from controls $(N=16)$. Predecidual cells showed intense immunostaining for VEGF-D (Fig. 4) but not VEGF-G (data not shown). There was a clear increase in VEGF-D immunostaining intensity in pre-decidual cells compared to non-decidualised stromal cells in areas where these two cell types appeared together (Fig. $4 A$ and $4 C$ ).

To further explore whether the process of decidualisation results in elevated expression of VEGF-C and VEGF-D protein, Western analysis was performed on human endometrial stromal cells before and after decidualisation in vitro ( $\mathrm{N}=5$ samples). Human endometrial stromal cells showed evidence by Western analysis for increased expression of both VEGF-C and VEGF-D after decidualisation in vitro (Fig. $5 A, 5 B$ and Fig. $\mathrm{S} 1$ ). Up to seven VEGF-D polypeptides were detected including 53, 44, 41, 29/31 and $21 \mathrm{kD}$ [24]. There was a significant increase in VEGF-D expression in decidualised versus non-decidualised cells $\left(\mathrm{F}_{(1,16)}=22.2, \quad P=0.001\right)$ with a significant variation among different isoforms $\left(\mathrm{F}_{(3,16)}=4.38, P=0.02\right)$. Paired T-tests were significant after Bonferoni correction for the $53 \mathrm{kD}$ (3.5-fold increase in relative density, $P=0.002)$ and $21 \mathrm{kD}(8.2$-fold increase in relative density, $P=0.005)$ isoforms, but not for the $41(P=0.05$, 


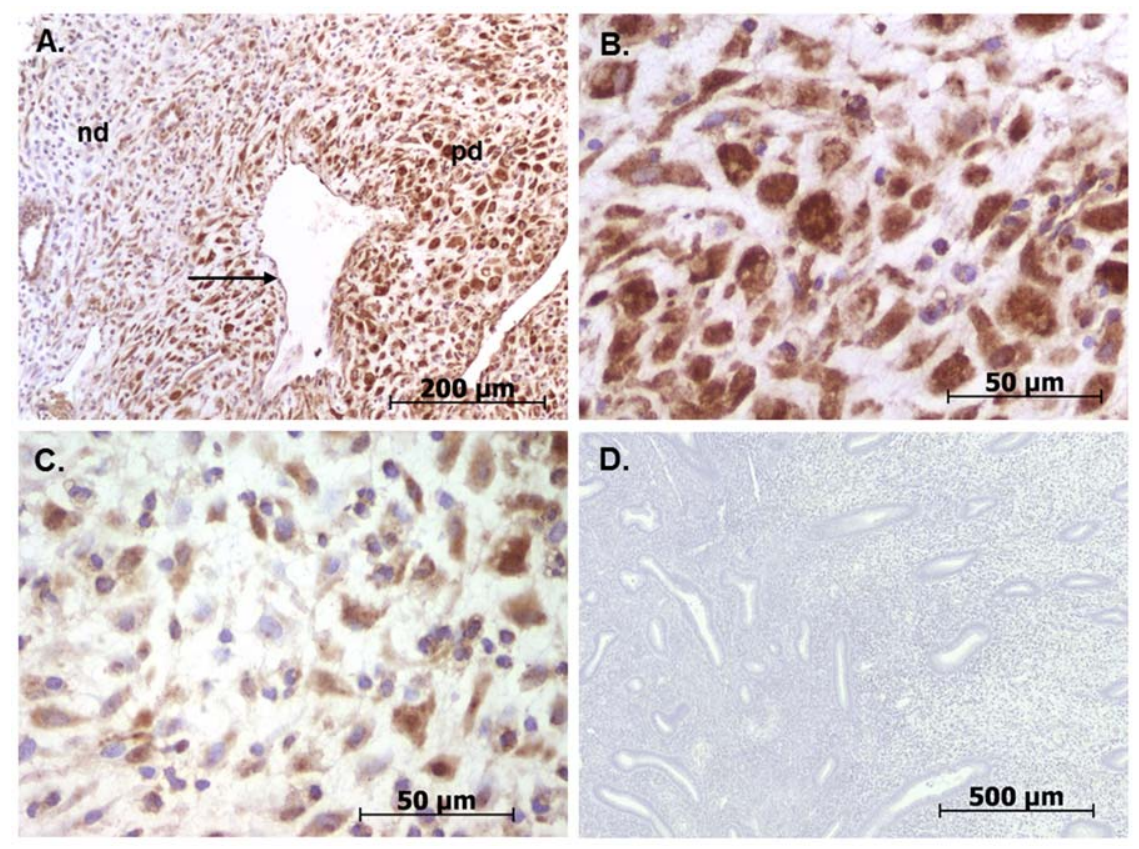

Figure 4. VEGF-D immunostaining in endometrial decidual cells. Distinct VEGF-D immunostaining (brown) is present in pre-decidual cells of endometrium from women treated with LNG-IUS prior to hysterectomy. (A) Micrograph illustrating difference in VEGF-D immunostaining (brown) intensity in stromal pre-decidualised cells ( $\mathrm{pd}$ ) compared to non-decidualised cells (nd). (B) Higher power image of pre-decidualised stromal cells showing VEGF-D immunostaining in brown. (C) Higher power image of non-decidualised stromal cells showing reduced VEGF-D immunostaining. (D) Isotype matched negative control. Black arrow: blood vessel. (sections are lightly counterstained with haematoxylin to identify cell nuclei in blue). doi:10.1371/journal.pone.0030916.g004

which fails to reach $P=0.0125$ as required by the Bonferoni correction) and $29 / 31 \mathrm{kD}(P=0.26)$ isoforms. The increase in the $21 \mathrm{kD}$ isoform is of particular relevance as following proteolytic processing this is the most biologically active isoform with the highest affinity for VEGF receptor-3 (VEGFR-3), which in the adult is found on lymphatic endothelial cells [25]. VEGF-C protein was detected as $58,43,29 / 31 \mathrm{kD}$ polypeptides [26]. Densitometry showed there was a significant increase in VEGF-C expression in decidualised versus non-decidualised cells by 2-way mixed ANOVA $\left(\mathrm{F}_{(1,16)}=25.7, P=0.001\right)$, as well as a significant variation among different isoforms $\left(\mathrm{F}_{(3,16)}=4.93, P=0.013\right)$. Paired T-tests for individual isoforms failed to reach significance after Bonferoni correction (Note: to reach significance after the Bonferoni correction, a P-value of 0.0125 is required: $58 \mathrm{KD}$ : $P=0.024$, 43 kD: $P=0.21,29 / 31 \mathrm{kD}: P=0.023 ; 21 \mathrm{kD}: \mathrm{P}=0.50$; Fig. 5C).

\section{Exogenous Progestin Produces a Pre-decidual Response in Human Endometrial Xenografts Resulting in VEGF-D Expression and Increased Lymphatic Vessel Diameters}

To further explore the role of exogenous progestin in causing lymphatic vessel dilatation through decidualisation-dependent expression of VEGF-D, a human endometrial xenograft model in NOD/scid mice was utilised. A total of $n=4$ human samples were subdivided and grafted into NOD/scid mice that were subsequently treated with estradiol valerate only, or estradiol valerate and medroxyprogesterone acetate (MPA). The estrogen component of this treatment maximizes xenograft establishment, vascularisation and survival, and the progestin recreates the hormonal status that exists in women using progestin contraceptives. There was a $100 \%$ recovery of graft tissue at the end of the experimental period. There were no obvious macroscopic differences between the treatment groups with all grafts becoming encapsulated by extracellular matrix. The endometrial vasculature was composed of both blood and lymphatic vessels which were predominantly of human origin $(92 \pm 3 \%$ ) with a small number of murine vessels also infiltrating the endometrial tissue $(8 \pm 3 \%)$ (Fig. 6A and 6B). All subsequent analysis of blood and lymphatic vessels only included vessels that exhibited positive immunostaining with human anti-CD31 antibody. The endometrial xenografts that received estrogen-only contained stromal cells that were densely packed and fibroblast-like in appearance while the glandular epithelium was cuboidal or columnar (Fig. 6C- H\&E staining). In contrast, the progestin-treated xenografts exhibited pre-decidualised stromal cells with flattened glandular epithelial cells (Fig. $6 D-\mathrm{H} \& \mathrm{E}$ staining). To confirm that decidual cells in progestin treated xenografts expressed VEGF-D, immunostaining was performed. VEGF-D immunostaining was intense in decidualised tissue in xenografts (Fig. 7), and was also apparent in epithelial cells.

Blood and lymphatic vessel density and cross sectional area were evaluated in progestin-treated $(n=4)$ and control xenografts $(\mathrm{n}=4)$ following immunostaining with antibodies to CD31 and D2-40. There was a significant increase in cross-sectional area of the 5 largest lymph vessels in the progestin treated xenografts $\left(4.6 \times 10^{-4} \pm 5.0 \times 10^{-5}\right.$ vessels $\left./ \mathrm{mm}^{2}\right)$ when compared to the estrogen-only treated xenografts $\left(1.1 \times 10^{-3} \pm 1.0 \times 10^{-4}\right.$ vessels/ $\left.\left.\mathrm{mm}^{2}\right) \mathrm{t}_{(6)}=-5.873, P=0.001\right)($ Fig. $8 D)$. Xenografts treated with progestin for 4 weeks $\left(245 \pm 35.9\right.$ vessels $\left./ \mathrm{mm}^{2}\right)$ had significantly reduced blood vessel density when compared to xenografts that received estrogen-only $\left(406 \pm 44.3\right.$ vessels $\left./ \mathrm{mm}^{2}\right)$ for the same length of time $\left(\mathrm{t}_{(6)}=2.848, P=0.029\right)$ (Fig. $8 A$ ). There was no difference in lymph vessel density between treatment groups (estrogen-only: $29 \pm 9.2$ vessels $/ \mathrm{mm}^{2}$; progestin: $23 \pm 3.1$ vessels/ $\left.\mathrm{mm}^{2} ; \mathrm{t}_{(6)}=0.619, P=0.559\right) \quad($ Fig. $8 B$ ). There was also no significant difference in the cross-sectional area of the 5 largest 


\section{A. VEGF-C}

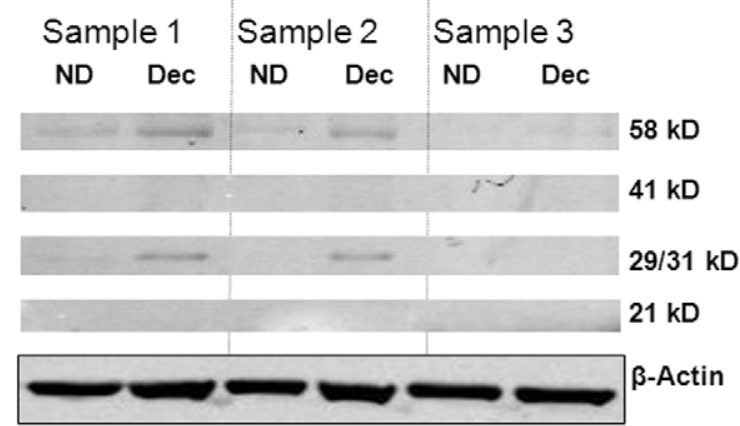

\section{B. VEGF-D}

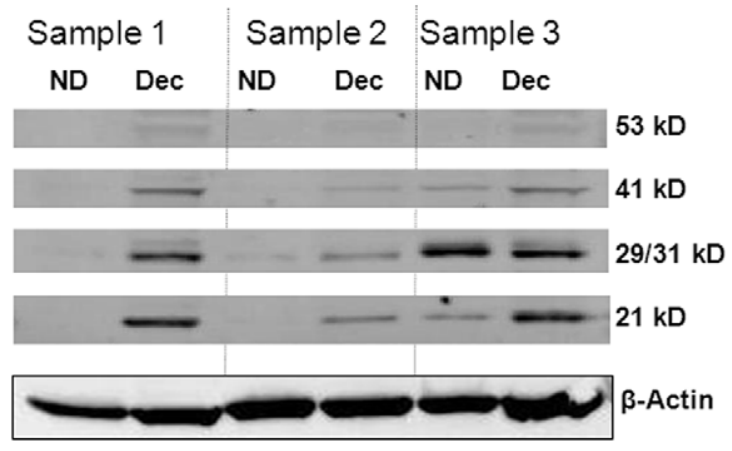

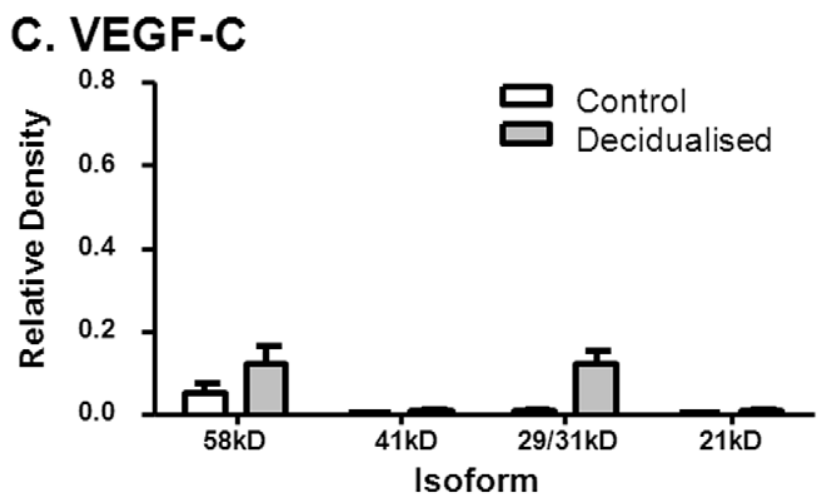

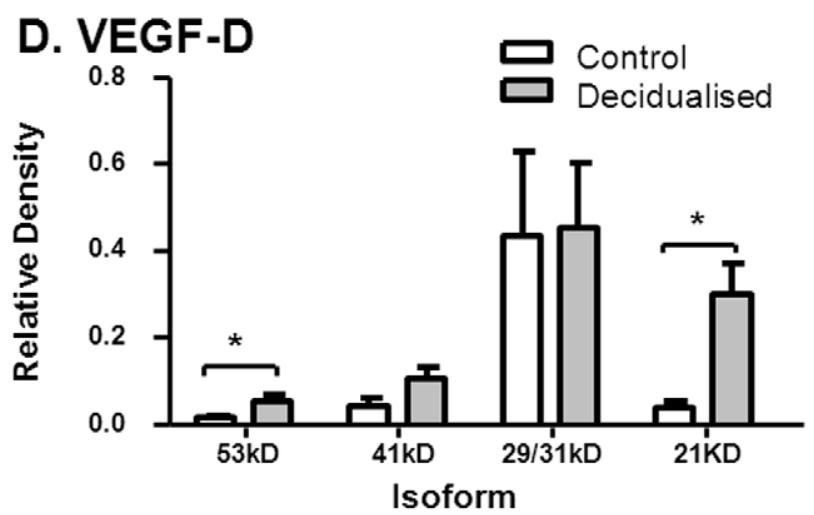

Figure 5. VEGF-D protein in endometrial decidual cells. Western analysis of vascular endothelial cell growth factor-C (VEGF-C) and VEGF-D peptide expression in primary cultures of human endometrial stromal cells (HESC). Panels A and B show representative data from 3 samples. Panels $C$ and D show combined densitometry data from all 5 samples. (A) VEGF-C peptide expression $(58,41,29 / 31$ and $21 \mathrm{kD}$ ) in HESC (ND) and decidualised HESC (Dec). (B) VEGF-D peptide expression (53, 41, 29/31 and 21 kD) in HESC and decidualised HESC. (C-D) Densitometry of VEGF-C and VEGF-D peptide expression. White bars representing control non-decidualised HESC and black bars representing decidualised HESC. Columns represent means $\pm \mathrm{SE}$; $P<0.05$

doi:10.1371/journal.pone.0030916.g005

blood vessels between the treatment groups (estrogen-only: $8.7 \times 10^{-4} \pm 3.0 \times 10^{-4} \quad$ vessels $/ \mathrm{mm}^{2} ; \quad$ progestin: $2.8 \times 10^{-3} \pm$ $1.1 \times 10^{-3}$ vessels $\left./ \mathrm{mm}^{2} ; \mathrm{t}_{(6)}=-1.681, P=0.179\right)($ Fig. $8 C$ ).

\section{Discussion}

In this study, we make the novel finding that the large, dilated vessels characteristic of progestin-treated endometrium include both blood and lymphatic vessels (Fig. 1). That both blood and lymphatic vessels exhibit abnormal morphology and dilatation in response to progestin is consistent with a regulatory mechanism common to both vessels types. Evidence for such a mechanism comes from studies evaluating the therapeutic potential of human VEGF family members using adenoviral gene transfer in rabbit hind limb skeletal muscle [23]. The VEGF family of angiogenic and lymphangiogenic peptides includes VEGF-A, VEGF-B, VEGF-C, VEGF-D and placental growth factor [27]. VEGF-C and VEGF-D act by binding and activating the tyrosine kinase receptors VEGF receptor-2 (VEGFR-2) and VEGFR-3, which in the adult are found predominantly on blood and lymphatic endothelial cells, respectively [28,29]. VEGF-C and VEGF-D differ from other members of the mammalian VEGF family due to the presence of pro-peptides at both the $\mathrm{N}$ - and C-termini of the conserved VEGF homology domain [26]. Cleavage of the pro-peptides by proteases such as plasmin modulates bioactivity of both molecules, increasing affinity for both VEGFR-2 and
VEGFR-3 [25,26,30]. In the rabbit hind limb skeletal muscle study, both VEGF-A and the proteolytically processed $21 \mathrm{kD}$ form of VEGF-D caused a remarkable enlargement of microvessels, however, while VEGF-A only affected blood vessels, VEGF-D enlarged both blood and lymph vessels [23]. VEGF-A also moderately increased capillary density, whereas VEGF-D induced angiogenesis was more diffuse. VEGF-D has also been shown to induce vessel enlargement in mouse hind limb [23,31], rat cremaster muscle [32], pig heart [33], mouse skin [34] and mouse uterine horn [35]. The remarkable similarity in the changes to the blood and lymph microvessels in our study and those previously published provided the impetus to further investigate a role for VEGF-D in the progestin treated human endometrium.

Progestin-treated endometrium usually exhibits areas of decidualisation, especially in the first few months of exposure before endometrial regression has occurred $[17,18,36]$. However, in contrast to the normal menstrual cycle, these decidual cells are maintained by the exogenous progestin for a relatively long period of time in the absence of implantation or placentation. Given that decidual cells play a central role in placentation, it is reasonable to speculate that the pathological vascular changes seen in progestinaffected endometrium are the consequence of normal vascular remodelling for placentation that has become defective through a lack of implantation. The observation that BTB occurs with nearly all types of progestin is consistent with the hypothesis that it is the 


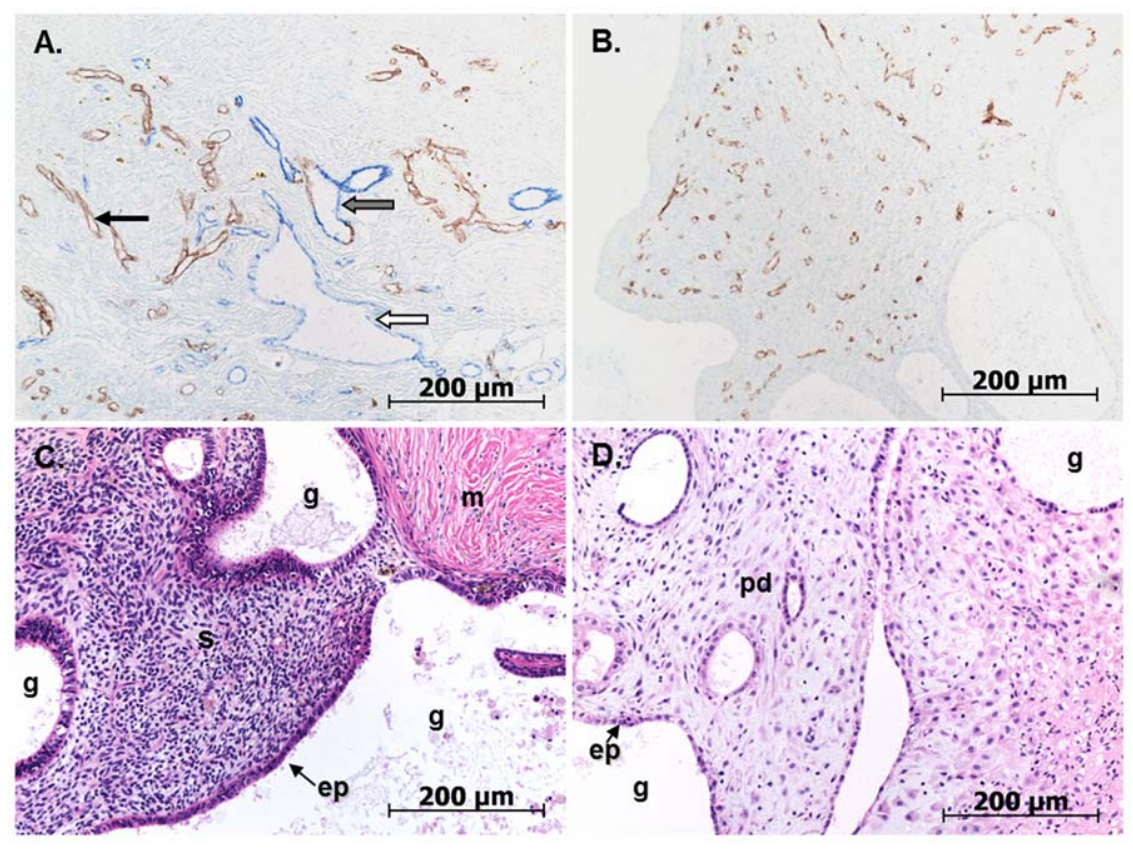

Figure 6. Human endometrial xenografts in NOD/scid mice. Representative micrographs of human endometrial xenografts taken from NOD/ scid mice after 6 weeks growth at a subcutaneous site. (A) A mixture of human and mouse vessels at the edge (capsule) of the xenograft shown by double immunostaining with anti-human CD31 (brown, black arrow) and anti-mouse CD31 (blue, white arrow). Note the vessel of mixed human and mouse origin (grey arrow). (B) The central portion of the xenograft populated almost exclusively with vessels of human origin (double immunostaining with anti-human CD31 in brown and anti-mouse CD31 in blue). (C) Routine haematoxylin and eosin stained xenografts treated with estradiol valerate developed densely packed fibroblast-like stromal cells and cuboidal to columnar epithelial cells. (D) Routine haematoxylin and eosin stained xenografts grafts treated with MPA displayed pre-decidualised stromal cells with flattened epithelial cells. ep: epithelium, g: glands, m: myometrium, pd: pre-decidual stroma, s: stroma.

doi:10.1371/journal.pone.0030916.g006

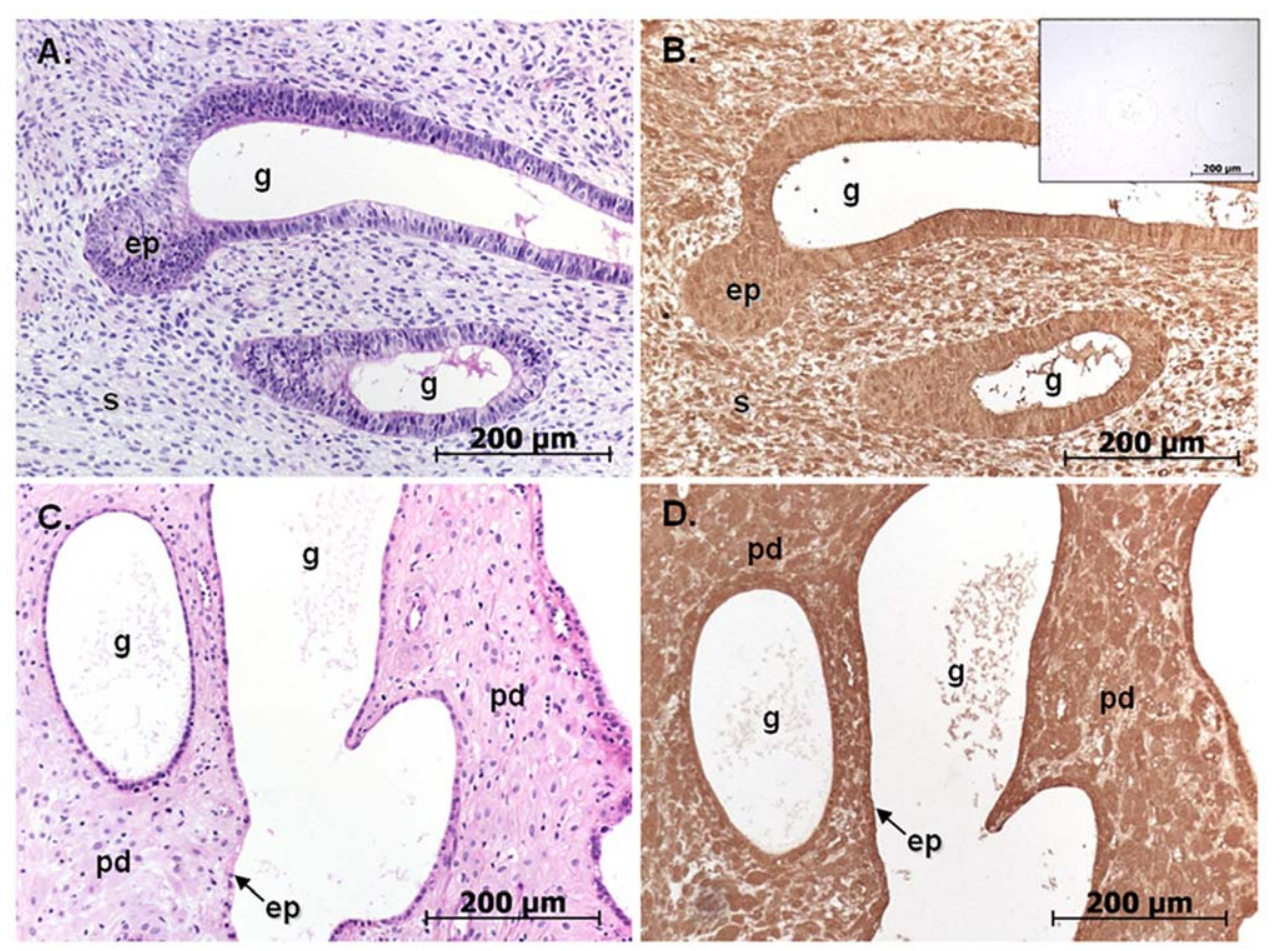

Figure 7. VEGF-D immunostaining of xenograft decidual cells. Representative micrographs of human endometrial xenografts from NOD/scid mice treated with estradiol valerate $(A, B)$ or medroxyprogesterone acetate $(C, D)$. Serial sections were stained with haematoxylin and eosin $(A, C)$ or immunostained with VEGF-D (B,D). Inset in (B): Isotype matched negative control. Note the pre-decidual cells in MPA-treated sections. ep: epithelium, g: glands, pd: pre-decidual stroma, s: stroma.

doi:10.1371/journal.pone.0030916.g007 

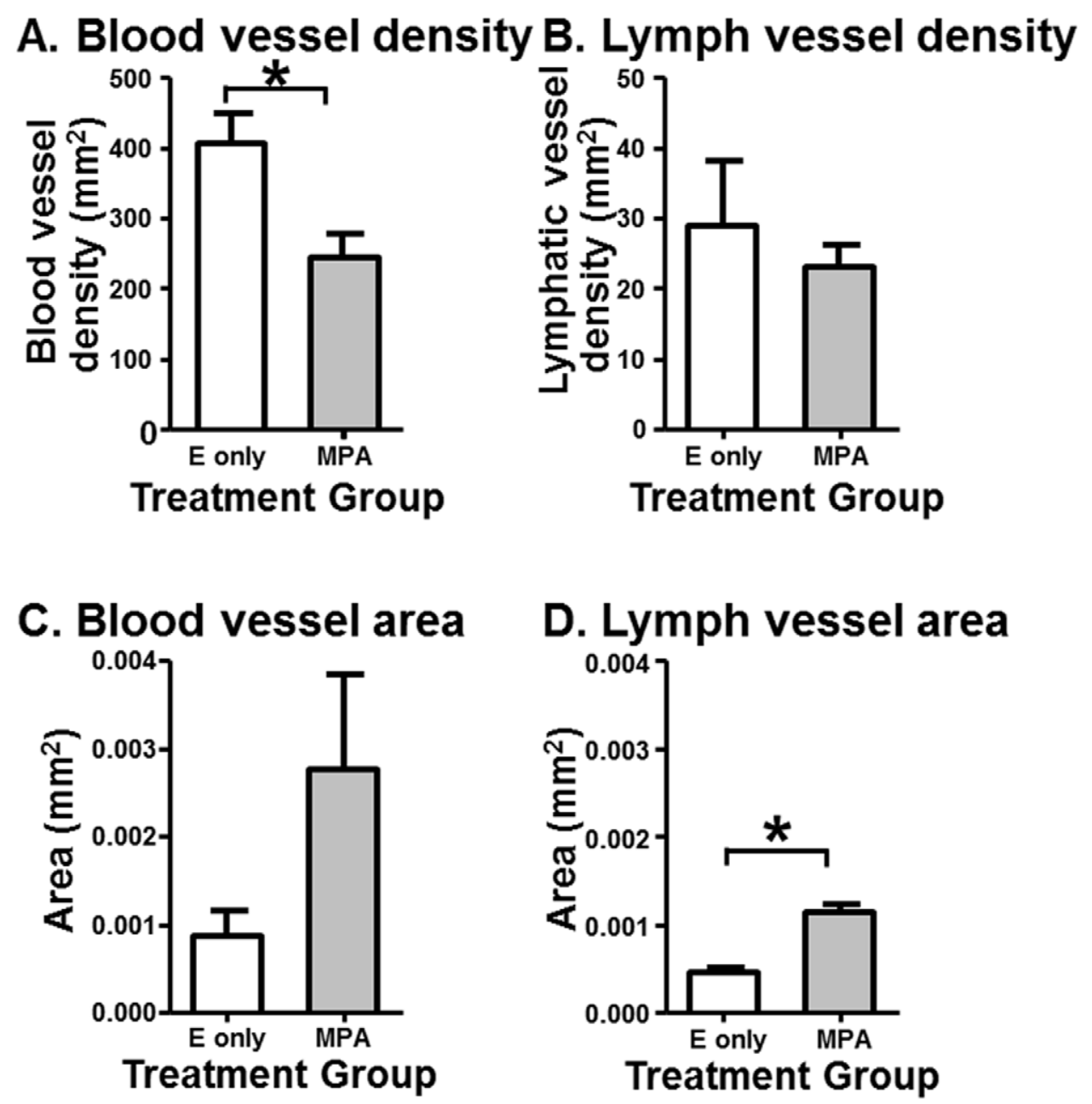

Figure 8. Progestin effects on xenograft vessel density and area. Blood vessel density decreases and lymphatic vessel cross sectional vessel area increases in human endometrial xenografts treated with medroxyprogesterone acetate (MPA). (A) Blood vessel density and (B) lymphatic vessel density of untreated (E only) and treated (MPA) xenografts. (C) Blood vessel and (D) lymphatic vessel cross sectional area of E only and MPA treated xenografts. Bars represent means $\pm \mathrm{SE}$. * $P<0.05$.

doi:10.1371/journal.pone.0030916.g008

prolonged presence of decidual cells that is causing BTB, rather than a direct effect of the progestins on blood vessels. In a mouse model of BTB it has been shown that endometrial breakdown following prolonged progestin treatment is dependent on decidualisation [37]. Although this study does not link decidualisation directly to a mechanism that causes vascular dilatation, it points towards a relationship between decidualisation and BTB.

While VEGF-D is a highly plausible candidate for causing the microvascular changes seen in progestin-exposed endometrium, decidual cells have upregulated expression of many other proteins, including connexin 43, prolactin, IL-6 and VEGF-A [38]. It is possible that one or several of these other agents may play contributory roles in causing BTB. We have previously investigated VEGF-A expression by immunohistochemistry in decidualised and non-decidualised endometrial stromal cells of women with BTB following progestin-only contraception and found no difference [39], despite the fact that it is up-regulated in in vitro decidualised stromal cells [38].

While the current study establishes a potential mechanistic link between progestins, decidualisation and endometrial microvessel dilatation, the link between dilated endometrial vessels and BTB remains more speculative. Further, if a relationship between dilated endometrial vessels and BTB does exist, we would assume that it is the blood vessels and not the lymphatic vessels that are the primary cause. There are several published studies that do support a link between vessel dilatation and BTB. Most significantly, dilated endometrial vessels, which are a common occurrence with systemic or intrauterine progestin exposure, have been shown to demonstrate increased fragility in hysteroscopic studies [13,19,40]. Additionally, it has been shown that perivascular $\alpha$-smooth muscle actin ( $\alpha \mathrm{SMA})$ is reduced around the endometrial vessels of progestin users with BTB compared to those with no bleeding problems [11]. Reduced $\alpha$ SMA indicates a loss of perivascular support cells, specifically pericytes and vascular smooth muscle cells, a process which could be expected to contribute significantly to increased vascular fragility and BTB. Other groups have also reported a reduction in endometrial vessel $\alpha$ SMA coverage following exposure to the progestin levonorgestrel [12]. This study suggested that time-dependent changes in blood vessel number, area, density and maturation following exposure to LNGIUS may explain the early transient increase in BTB. In a further study examining the link between BTB and endometrial vessel structure, it was reported that in women with BTB after 12 months of progestin treatment, significantly greater numbers of enlarged, 
distended vessels were present in tissues taken from bleeding sites compared with non-bleeding sites [41]. These authors suggested that chronic over expression of endometrial Tissue Factor (TF) at the bleeding sites might be promoting aberrant angiogenesis, resulting in the enlarged and fragile vessels with increased risk of bleeding.

In the current study we used a xenograft model to investigate the progestin-related changes to human endometrium caused by medroxyprogesterone acetate treatment in vivo. Our results parallel a recent study that used LNG to treat human endometrial xenografts for 4 weeks in SCID mice [42]. In both studies, 4 weeks of progestin exposure caused heterogeneous decidualisation and an increase in vessel diameter in subcutaneous endometrial explants. Additionally, both models retained a greater than $90 \%$ presence of human vessels within the xenograft with minimal invasion of host vasculature [43]. The similar observations from these 2 independent studies confirm the utility of the xenograft model for studying human endometrial microvascular function.

The current study identifies both decidual cells and VEGF-D as potential targets for development of therapies for BTB. If decidual derived VEGF-D is the agent responsible for BTB, then a number of interventions might be possible. These include developing progestins that minimise or avoid decidualisation, developing agents that block progestin-induced decidualisation, and using inhibitors of VEGF-D to prevent endometrial microvascular dilatation.

Despite BTB having been a significant clinical problem for many years, advances in understanding the mechanisms that cause BTB have been limited. The lack of a readily available animal model that reliably replicates progestin-induced BTB is probably the single most critical issue responsible for this lack of progress. The absence of an accepted model means that mechanistic studies can only be performed in humans, which limits significantly the research that can be undertaken. The xenograft model used the current study does not exhibit BTB, despite replicating many of the changes seen in human endometrium exposed to progestins. Future progress in understanding BTB is most likely to come from a combination of indirect mechanistic studies supported by observational and correlational data from human subjects with BTB.

In conclusion, we have identified dilated lymphatics in progestin exposed human endometrium from women with BTB, which in conjunction with dilated blood vessels identifies VEGF-D as a potential causative agent. Further studies show that VEGF-D expression increases as endometrial stromal cells undergo decidualisation, and that this chain of events can be replicated in human endometrial xenografts in NOD/scid mice treated with progestin. Taken together these data implicate decidual cellderived VEGF-D as a causative factor in progestin-induced BTB.

\section{Materials and Methods}

\section{Human samples and specimen collection}

Fixed and wax embedded human uterine tissue blocks from a previously published study [22] were obtained from women that had been exposed to LNG-IUS as treatment for heavy menstrual bleeding. Briefly, women who opted for a hysterectomy due to continued heavy menstrual bleeding following standard treatment options, were randomly allocated into LNG-IUS $(n=16$, mean age 38.6 years) or control groups $(n=16$, mean age 39.9 years). LNGIUS were in place for up to 156 days prior to surgery. For the xenograft study, uterine biopsies were obtained following written informed consent (this work was approved by Southern Health Human Research and Ethics Committee under Project
No. 08176B) from 4 patients aged $48.7 \pm 1$ year undergoing hysterectomy for prolaspe or fibroids. Each sample was cut down to the endometrial myometrial boarder and divided into 8 grafts approximately $1 \times 1 \times 0.5 \mathrm{~cm}$ in size.

\section{Animals and Hormone Treatments}

For xenograft studies, NOD/SCID female mice $(n=32 ; 6-8$ weeks) received a single graft in a pocket created on the right side of the back flank (This work was carried out in accordance with the Australian Code of Practice for the Care and Use of Animals for Scientific Purposes (2004) and approved by Monash Medical Centre Animal Ethics Committee A, approval No. 2008/14). Following closure of the wound site, mice received $1 \mu \mathrm{g} 17 \beta$ estradiol valerate in oil (subcutaneous) every fourth day until experiment completion. After two weeks of graft establishment, mice were divided into two groups and received a subcutaneous silastic tube implant [44]. The $17 \beta$-estradiol valerate group (Control) received an empty implant while the medroxyprogesterone acetate (MPA-Progestin) group received an implant containing $20 \mathrm{mg}$ of MPA. Progestin treatment was continued for 4 weeks until graft harvest and fixation.

\section{Sample collection and Immunohistochemistry}

Patient samples and xenografts were formalin fixed and paraffin embedded for immunohistological analysis. Tissue sections $(5 \mu \mathrm{m})$ mounted on silane coated slides were dewaxed and rehydrated. Following antigen retrieval, endogenous peroxide block and protein block, immunolocalisation of human vascular endothelial cells (CD31: Clone JC 70A, Dako Cytomation, CA, USA,), human lymphatic endothelial cells (anti-Podoplanin: Clone D2-40, Signet Laboratories, MA, USA) [45], mouse vascular endothelial cells (CD31: \#553370, BD Pharmingen, NJ, USA) and human VEGFD (\#AF469, R\&D Systems MN USA) was performed as previously described [35]. Using a $20 \times$ objective lens, the five largest vessels within the endometrial component of the tissue were identified and measured in $\mathrm{mm}^{2}$ using the Analytical Imaging System (AIS) (Imaging Research Inc. GE Healthcare Bio-Sciences, NSW, Australia). We found this approach to be a simple and statistically robust morphometric method for objectively demonstrating the existence of the abnormally dilated and thin-walled vessels found in progestin-exposed endometrium.In xenograft samples, vessel density was determined by calculating the average number of vessels from 4 separate endometrial areas. Statistical analysis was performed using SPSS for Windows, Version 11.0.0 (SPSS Inc., Chicago, IL, USA). Vessel cross sectional area and vessel density of the functional layer and basal layer vessels in the endometrium of women +/ - LNG-IUS was determined by 2-way ANOVA on logged data followed by Tukeys HSD post hoc analysis. Statistical analysis of xenograft $+/-$ MPA vessel density and diameter was determined by Student $t$ test. Values are presented as mean $\pm \mathrm{SE}$ unless otherwise stated. A p value $<0.05$ was considered significant.

\section{Western Blotting}

Primary isolated human endometrial stromal cells (from $n=5$ endometrial biopsies) were decidualised following incubation with estradiol $17 \beta\left(10^{-8} \mathrm{~mol} / \mathrm{l}\right)$ and progesterone $\left(10^{-7} \mathrm{~mol} / \mathrm{l}\right)$ as previously described [46]. Protein from cell lysates $(30 \mu \mathrm{g})$ were resolved on a 4-12\% NuPAGE gel (Invitrogen Corporation; CA, USA). Following transfer to PVDF membranes, proteins were probed for VEGF-C (1:200; AF286 R\&D Systems; MN, USA), VEGF-D (1:200; AF752 R\&D Systems) or $\beta$-actin (1:4000; Sigma Aldrich; NSW, Australia) overnight at $4^{\circ} \mathrm{C}$. Signal was enhanced with fluorescent secondary antibodies (1:20,000; AlexaFluor 680 
Invitrogen or IRD 800 LI-COR Biosciences; NE, USA). Membranes were scanned and densitometry determined by the Odyssey ${ }^{\circledR}$ Infrared Imaging System (LI-COR Biosciences). The relative densitometry for each peptide band was normalised to the $\beta$-actin and expressed as a percentage of $\beta$-actin. Densitometry data from VEGF-C and VEGF-D western blots were analysed using a 2-way Mixed ANOVA followed by posthoc analysis using paired T-tests with Bonferoni corrections to correct for multiple comparisons.

\section{Supporting Information}

Figure S1 Representative western blots of (A) VEGF-C and (B) VEGF-D protein expression in primary cultures of decidualised (Dec) and non-decidualised (ND) human endometrial stromal cells. White arrows indicate the reported isoforms including the $\mathrm{C}$ VHD-N full length receptor (VEGF-C: $58 \mathrm{kD}$; VEGF-D: $53 \mathrm{kD}$;

\section{References}

1. Rodriguez MI, Warden M, Darney PD (2010) Intrauterine progestins, progesterone antagonists, and receptor modulators: a review of gynecologic applications. Am J Obstet Gynecol 202: 420-428.

2. Stewart A, Cummins C, Gold L, Jordan R, Phillips W (2001) The effectiveness of the levonorgestrel-releasing intrauterine system in menorrhagia: a systematic review. BJOG 108: 74-86.

3. Rodgers AK, Falcone T (2008) Treatment strategies for endometriosis. Expert Opin Pharmacother 9: 243-255.

4. d'Arcangues C (2000) Management of vaginal bleeding irregularities induced by progestin-only contraceptives. Hum Reprod 15 Suppl 3: 24-29.

5. Findlay JK (1996) Future directions for research on endometrial bleeding. Hum Reprod 11 Suppl 2: 179-183.

6. Markee JE (1978) Menstruation in intraocular endometrial transplants in the Rhesus monkey. Am J Obstet Gynecol 131: 558-559.

7. Rogers PA (1996) Endometrial vasculature in Norplant users. Hum Reprod 11 Suppl 2: 45-50.

8. Wonodirekso S, Affandi B, Siregar B, Barasila AC, Damayanti L, et al. (2000) Endometrial epithelial integrity and subepithelial reticular fibre expression in progestin contraceptive acceptors. Hum Reprod 15 Suppl 3: 189-196.

9. Vincent AJ, Zhang J, Ostor A, Rogers PA, Affandi B, et al. (2002) Decreased tissue inhibitor of metalloproteinase in the endometrium of women using depot medroxyprogesterone acetate: a role for altered endometrial matrix metalloproteinase/tissue inhibitor of metalloproteinase balance in the pathogenesis of abnormal uterine bleeding? Hum Reprod 17: 1189-1198.

10. Clark DA, Wang S, Rogers P, Vince G, Affandi B (1996) Endometrial lymphomyeloid cells in abnormal uterine bleeding due to levonorgestrel (Norplant). Hum Reprod 11: 1438-1444.

11. Rogers PA, Plunkett D, Affandi B (2000) Perivascular smooth muscle alpha-actin is reduced in the endometrium of women with progestin-only contraceptive breakthrough bleeding. Hum Reprod 15 Suppl 3: 78-84.

12. Stephanie R, Labied S, Blacher S, Frankenne F, Munaut G, et al. (2007) Endometrial vessel maturation in women exposed to levonorgestrel-releasing intrauterine system for a short or prolonged period of time. Hum Reprod 22: 3084-3091.

13. Hickey M, Dwarte D, Fraser IS (2000) Superficial endometrial vascular fragility in Norplant users and in women with ovulatory dysfunctional uterine bleeding. Hum Reprod 15: 1509-1514.

14. Wulff C, Weigand M, Kreienberg R, Fraser HM (2003) Angiogenesis during primate placentation in health and disease. Reproduction 126: 569-577.

15. Volchek M, Girling JE, Lash GE, Cann L, Kumar B, et al. (2010) Lymphatics in the human endometrium disappear during decidualization. Hum Reprod 25: 2455-2464.

16. Critchley HO, Wang H, Kelly RW, Gebbie AE, Glasier AF (1998) Progestin receptor isoforms and prostaglandin dehydrogenase in the endometrium of women using a levonorgestrel-releasing intrauterine system. Hum Reprod 13: 1210-1217.

17. Rogers PA, Au CL, Affandi B (1993) Endometrial microvascular density during the normal menstrual cycle and following exposure to long-term levonorgestrel. Hum Reprod 8: 1396-1404.

18. Song JY, Fraser IS (1995) Effects of progestogens on human endometrium. Obstet Gynecol Surv 50: 385-394.

19. Hickey M, Fraser I, Dwarte D, Graham S (1996) Endometrial vasculature in Norplant users: preliminary results from a hysteroscopic study. Hum Reprod 11 Suppl 2: 35-44.

20. Irvine GA, Campbell-Brown MB, Lumsden MA, Heikkila A, Walker JJ, et al. (1998) Randomised comparative trial of the levonorgestrel intrauterine system and norethisterone for treatment of idiopathic menorrhagia. $\mathrm{Br} \mathrm{J}$ Obstet Gynaecol 105: 592-598. dimers at $105 \mathrm{kD}$ ), the VHD-N terminal (doublet, 29/31 kD), and the VHD $(21 \mathrm{kD})$. Other bands may be alternatively processed forms including heterodimers, trimers and dimers, some of which also exhibit changes associated with decidualisation (black arrows). (TIF)

\section{Acknowledgments}

Thanks are due to Julia Rubulis for help with Westerns and Francis Walker for tissue collection.

\section{Author Contributions}

Conceived and designed the experiments: JFD CJM JEG PAWR. Performed the experiments: JFD CJM FLL LMC LF. Analyzed the data: JFD CJM JEG. Contributed reagents/materials/analysis tools: ED. Wrote the paper: JFD JEG PAWR.

21. Fraser IS, Hickey M (2000) Endometrial vascular changes and bleeding disturbances with long-acting progestins. Steroids 65: 665-670.

22. McGavigan CJ, Dockery P, Metaxa-Mariatou V, Campbell D, Stewart CJ, et al. (2003) Hormonally mediated disturbance of angiogenesis in the human endometrium after exposure to intrauterine levonorgestrel. Hum Reprod 18: 77-84.

23. Rissanen TT, Markkanen JE, Gruchala M, Heikura T, Puranen A, et al. (2003) VEGF-D is the strongest angiogenic and lymphangiogenic effector among VEGFs delivered into skeletal muscle via adenoviruses. Circ Res 92: 1098-1106.

24. Stacker SA, Caesar C, Baldwin ME, Thornton GE, Williams RA, et al. (2001) VEGF-D promotes the metastatic spread of tumor cells via the lymphatics. Nat Med 7: 186-191.

25. Stacker SA, Stenvers K, Caesar C, Vitali A, Domagala T, et al. (1999) Biosynthesis of vascular endothelial growth factor-D involves proteolytic processing which generates non-covalent homodimers. J Biol Chem 274: 32127-32136.

26. Joukov V, Sorsa T, Kumar V, Jeltsch M, Claesson-Welsh L, et al. (1997) Proteolytic processing regulates receptor specificity and activity of VEGF-C. EMBO J 16: 3898-3911.

27. Adams RH, Alitalo K (2007) Molecular regulation of angiogenesis and lymphangiogenesis. Nat Rev Mol Cell Biol 8: 464-478.

28. Veikkola T, Karkkainen M, Claesson-Welsh L, Alitalo K (2000) Regulation of angiogenesis via vascular endothelial growth factor receptors. Cancer Res 60: 203-212.

29. Kaipainen A, Korhonen J, Mustonen T, van Hinsbergh VW, Fang GH, et al. (1995) Expression of the fms-like tyrosine kinase 4 gene becomes restricted to lymphatic endothelium during development. Proc Natl Acad Sci U S A 92: 3566-3570

30. McColl BK, Baldwin ME, Roufail S, Freeman C, Moritz RL, et al. (2003) Plasmin activates the lymphangiogenic growth factors VEGF-C and VEGF-D. J Exp Med 198: 863-868.

31. Kholova I, Koota S, Kaskenpaa N, Leppanen P, Narvainen J, et al. (2007) Adenovirus-mediated gene transfer of human vascular endothelial growth factor$\mathrm{d}$ induces transient angiogenic effects in mouse hind limb muscle. Hum Gene Ther 18: 232-244.

32. Byzova TV, Goldman CK, Jankau J, Chen J, Cabrera G, et al. (2002) Adenovirus encoding vascular endothelial growth factor-D induces tissue-specific vascular patterns in vivo. Blood 99: 4434-4442.

33. Rutanen J, Rissanen TT, Markkanen JE, Gruchala M, Silvennoinen P, et al. (2004) Adenoviral catheter-mediated intramyocardial gene transfer using the mature form of vascular endothelial growth factor-D induces transmural angiogenesis in porcine heart. Circulation 109: 1029-1035.

34. Veikkola T, Jussila L, Makinen T, Karpanen T, Jeltsch M, et al. (2001) Signalling via vascular endothelial growth factor receptor-3 is sufficient for lymphangiogenesis in transgenic mice. EMBO J 20: 1223-1231.

35. Girling JE, Donoghue JF, Lederman FL, Cann LM, Achen MG, et al. (2010) Vascular endothelial growth factor-D over-expressing tumor cells induce differential effects on uterine vasculature in a mouse model of endometrial cancer. Reprod Biol Endocrinol 8: 84.

36. Jones RL, Critchley HO (2000) Morphological and functional changes in human endometrium following intrauterine levonorgestrel delivery. Hum Reprod 15 Suppl 3: 162-172.

37. Morison NB, Zhang J, Kaitu'u-Lino TJ, Fraser IS, Salamonsen LA (2007) The long-term actions of etonogestrel and levonorgestrel on decidualized and nondecidualized endometrium in a mouse model mimic some effects of progestogenonly contraceptives in women. Reproduction 133: 309-321.

38. Yu J, Wu J, Bagchi IC, Bagchi MK, Sidell N, et al. (2011) Disruption of gap junctions reduces biomarkers of decidualization and angiogenesis and increases 
inflammatory mediators in human endometrial stromal cell cultures. Mol Cell Endocrinol 344: 25-34.

39. Rogers PA, Martinez F, Girling JE, Lederman F, Cann L, et al. (2005) Influence of different hormonal regimens on endometrial microvascular density and VEGF expression in women suffering from breakthrough bleeding. Hum Reprod 20: 3341-3347.

40. Hickey M, Dwarte D, Fraser IS (1998) Precise measurements of intrauterine vascular structures at hysteroscopy in menorrhagia and during Norplant use. Hum Reprod 13: 3190-3196.

41. Runic R, Schatz F, Wan L, Demopoulos R, Krikun G, et al. (2000) Effects of norplant on endometrial tissue factor expression and blood vessel structure. J Clin Endocrinol Metab 85: 3853-3859.
42. Alvarez Gonzalez ML, Galant C, Frankenne F, Nisolle M, Labied S, et al. (2009) Development of an animal experimental model to study the effects of levonorgestrel on the human endometrium. Hum Reprod 24: 697-704.

43. Alvarez Gonzalez ML, Frankenne F, Galant C, Marbaix E, Foidart JM, et al. (2009) Mixed origin of neovascularization of human endometrial grafts in immunodeficient mouse models. Hum Reprod 24: 2217-2224.

44. Cohen PE, Milligan SR (1993) Silastic implants for delivery of oestradiol to mice. J Reprod Fertil 99: 219-223.

45. Donoghue JF, Lederman FL, Susil BJ, Rogers PA (2007) Lymphangiogenesis of normal endometrium and endometrial adenocarcinoma. Hum Reprod 22: 1705-1713.

46. Menkhorst E, Salamonsen LA, Zhang J, Harrison CA, Gu J, et al. (2010) Interleukin 11 and activin A synergise to regulate progesterone-induced but not cAMP-induced decidualization. J Reprod Immunol 84: 124-132. 